\title{
Vector Variational-Like Inequalities with Generalized Semimonotone Mappings
}

\author{
Suhel Ahmad Khan \\ Department of Mathematics, BITS Pilani, Dubai Campus, 345055 Dubai, UAE \\ Correspondence should be addressed to Suhel Ahmad Khan; khan.math@gmail.com
}

Received 14 August 2012; Accepted 20 October 2012

Academic Editor: Ying $\mathrm{Hu}$

Copyright (C) 2013 Suhel Ahmad Khan. This is an open access article distributed under the Creative Commons Attribution License, which permits unrestricted use, distribution, and reproduction in any medium, provided the original work is properly cited.

\begin{abstract}
We introduce the concepts of generalized relaxed monotonicity and generalized relaxed semimonotonicity. We consider a class of generalized vector variationa-llike inequality problem involving generalized relaxed semimonotone mapping. By using KakutaniFan-Glicksberg's fixed-point theorem, we prove the solvability for this class of vector variational-like inequality with relaxed monotonicity assumptions. The results presented in this paper generalize some known results for vector variational inequality in recent years.
\end{abstract}

\section{Introduction}

Vector variational inequalities were initially introduced and considered by Giannessi [1] in a finite-dimensional Euclidean space in 1980. Due to its wide application the theory of the vector variational inequality is generalized in different directions and many existence results and algorithms for vector variational inequality problems have been established under various conditions; see for examples [2-7] and references therein.

The concept monotonicity and the compactness operators are very useful in nonlinear functional analysis and its applications. In 1968, Browder [8] first combined the compactness and accretion of operators and posed the concept of a semiaccretive operator. Motivated by this idea, Chen [9] studied the concept of a semimonotone operator, which combines the compactness and monotonicity of an operator and posed it to the study of variational inequalities. Recently in 2003, Fang and Huang [10] introduced relaxed $\eta$ - $\alpha$-semimonotone mapping, a generalized concept of semimonotonicity, and they established several existence results for the variational-like inequality problem.

In this paper, we pose two new concepts of generalized relaxed monotonicity and generalized relaxed semimonotonicity as well as two classes of generalized vector variational-like inequalities with generalized relaxed monotone mappings and generalized relaxed semimonotone mappings. We investigate the solvability of vector variationallike inequalities with generalized relaxed semimonotone mappings by means of the Kakutani-Fan-Glicksberg fixedpoint theorem. The results presented in this paper generalize the results of Chen [9], Fang and Huang [10], Usman and Khan [11], and Zheng [7].

\section{Preliminaries}

Throughout the paper unless otherwise specified, let $X$ and $Y$ be two real Banach spaces, $K \subset X$ be a nonempty closed and convex subset of $X . P$ is said to be a closed convex and pointed cone with its apex at the origin, if the following conditions hold:

(i) $\lambda P \subset P$, for all $\lambda>0$,

(ii) $P+P \subset P$,

(iii) $P \cap(-P)=\{0\}$.

The partial order $\leq_{P}$ in $Y$, induced by the pointed cone $P$, is defined by declaring $x \leq_{P} y$ if and only if $y-x \in P$ for all $x, y$ in $Y$. An ordered Banach space is a pair $(Y, P)$ with the partial order induced by $P$. The weak order $\leq_{\text {int } P}$ 
in an ordered Banach space $(Y, P)$ with int $P \neq \varnothing$ is defined as $x \leq_{\text {int } P} y$ if and only if $y-x \in \operatorname{int} P$ for all $x, y$ in $Y$, where int $P$ denotes the interior of $P$. Let $L(X, Y)$ denote the space of all continuous linear mappings from $X$ into $Y$. A set-valued mapping $P: K \rightarrow 2^{Y}$ be such that for each $x \in K, P(x)$ is a proper, closed, convex cone with int $P(x) \neq \varnothing$ and let $P_{-}=\bigcap_{x \in K} P(x)$.

First, we give the concept of generalized relaxed monotone mapping. In order to do so, the definition of a vector monotone mapping is needed, which was posed by Chen [9].

Definition 1 (see [9]). Let $T: K \rightarrow L(X, Y)$ be a mapping, $K \subset X$ be a nonempty, closed and convex subset in $X$. Let $P$ : $K \rightarrow 2^{Y}$ be such that for each $x \in K, P(x)$ is a proper, closed, convex cone with int $P(x) \neq \varnothing$. T is said to be $P_{-}$-monotone on $K$ if and only if it satisfies the following condition:

$$
\langle T(y)-T(x), y-x\rangle \geq_{P_{-}} 0, \quad \forall x, y \in K,
$$

where $P_{-}=\bigcap_{x \in K} P(x)$.

We now give the concept of generalized relaxed monotone mapping.

Definition 2. Let $P: K \rightarrow 2^{Y}$ be such that for each $x \in$ $K, P(x)$ is a proper, closed, convex cone with int $P(x) \neq \varnothing$ and a mapping $\eta: K \times K \rightarrow X$. A mapping $T: K \rightarrow L(X, Y)$ is said to be generalized relaxed monotone, if

$$
\langle T y-T x, \eta(y, x)\rangle-\alpha(x, y) \geq_{P_{-}} 0, \quad \forall x, y \in K,
$$

where $\alpha: X \times X \rightarrow Y$ is a mapping such that $\lim _{t \rightarrow 0^{+}}(\alpha(x, t y+(1-t) x) / t)=0$.

Remark 3. (i) If $\alpha(x, y)=\alpha(x-y)$, where $\alpha: X \rightarrow \mathbb{R}$ with $\alpha(\lambda z)=\lambda^{p} \alpha(z)$ for $\lambda>0, p>1$, then inclusion (2)

$$
\langle T y-T x, \eta(y, x)\rangle-\alpha(x, y) \geq_{P_{-}} 0, \quad \forall x, y \in K
$$

reduces to

$$
\langle T y-T x, \eta(y, x)\rangle-\alpha(x-y) \in P_{-}, \quad \forall x, y \in K .
$$

Also if $P(x)=\mathbb{R}_{+}$, for all $x \in K$, then $P_{-}=\bigcap_{x \in K} P(x)=\mathbb{R}_{+}$. As a result the above inclusion reduces to

$$
\begin{aligned}
& \langle T y-T x, \eta(y, x)\rangle-\alpha(x-y) \geq 0, \quad \forall x, y \in K, \\
& \Longrightarrow\langle T y-T x, \eta(y, x)\rangle \geq \alpha(x-y), \quad \forall x, y \in K,
\end{aligned}
$$

then $T$ is said to be relaxed $\eta$ - $\alpha$-monotone, introduced and studied by Fang and Huang [10].

(ii) In the above inclusion (5), if we take $\eta(y, x)=y-$ $x$, for all $x, y \in K$, then it reduces to

$$
\langle T y-T x, y-x\rangle \geq \alpha(x-y), \quad \forall x, y \in K,
$$

then $T$ is said to be $\alpha$-relaxed monotone.

(iii) In the above inclusion (6), if we take $\alpha(z)=k\|z\|^{p}$, where $k>0$ is constant, then it reduces to

$$
\langle T y-T x, y-x\rangle \geq k\|y-x\|^{p}, \quad \forall x, y \in K .
$$

Then $T$ is called $p$-monotone, see for example [12-14].
We recall the following concepts and results which are needed in the sequel.

Definition 4. A mapping $f: K \rightarrow Y$ is said to be

(i) $P_{-}$-convex, if $f(t x+(1-t) y) \leq_{P_{-}} t f(x)+(1-$ t) $f(y)$, for all $x, y \in K, t \in[0,1]$;

(ii) $P_{-}$-concave, if $-f$ is $P_{-}$-convex.

Definition 5. A mapping $\eta: K \times K \rightarrow X$ is said to be affine in the first argument if for any $x_{i} \in K$ and $\lambda_{i} \geq 0,(1 \leq i \leq n)$, with $\sum_{i=1}^{n} \lambda_{i}=1$ and any $y \in K$,

$$
\eta\left(\sum_{i=1}^{n} \lambda_{i} x_{i}, y\right)=\sum_{i=1}^{n} \lambda_{i} \eta\left(x_{i}, y\right) .
$$

Lemma 6 (see [3]). Let $(Y, P)$ be an ordered Banach space with a proper, closed, convex and solid cone $P(x)$ then for all $x, y, z \in Y$, one has
(i) $z \Varangle_{\text {int } P(x)} x$ and $x \geq_{P(x)} y \Rightarrow z \Varangle_{\text {int } P(x)} y$,
(ii) $z \underline{\operatorname{tint}}_{P(x)} x$ and $x \leq_{P(x)} y \Rightarrow z \underline{\operatorname{tint}}_{P(x)} y$.

Lemma 7 (see [15]). Let $K$ be a subset of a topological vector space $X$ and let $F: K \rightarrow 2^{X}$ be a KKM mapping. If for each $x \in K, F(x)$ is closed and for at least one $x \in K, F(x)$ is compact, then

$$
\bigcap_{x \in K} F(x) \neq \varnothing
$$

Definition 8. A mapping $T: K \rightarrow L(X, Y)$ is said to be $\eta$-hemicontinuous, if for any $x, y \in K$, the mapping $t \rightarrow$ $\langle T x+t(y-x), \eta(y, x)\rangle$ is continuous at $0^{+}$.

Now, we have the following Minty's type Lemma.

Lemma 9. Let $K \subset X$ be a nonempty, closed, and convex subset of $X$. Let $P: K \rightarrow 2^{Y}$ be such that for each $x \in$ $K, P(x)$ is a proper, closed, convex cone with int $P(x) \neq \varnothing$. Let $f: K \times K \rightarrow Y$ be $P_{-}$-convex in the first argument with the condition $f(x, x)=0$, for all $x \in K$. Suppose the following conditions hold:

(i) $\eta: K \times K \rightarrow X$ is an affine in the first argument with the condition $\eta(x, y)+\eta(y, x)=0$, for all $x, y \in K$;

(ii) the set-valued mapping $W: K \rightarrow 2^{Y}$ defined as $W(x)=Y \backslash\{-\operatorname{int} P(x)\}$ for all $x \in K$ is weakly upper semicontinuous;

(iii) $T: K \rightarrow L(X, Y)$ is $\eta$-hemicontinuous and generalized relaxed monotone mapping.

Then the following two problems are equivalent:

(A) $x \in K,\langle T x, \eta(y, x)\rangle+f(y, x) \Varangle_{\text {int } P(x)} 0$, for all $y \in K$,

(B) $x \in K,\langle T y, \eta(y, x)\rangle+f(y, x)-\alpha(x, y)$ $\$_{\text {int } P(x)} 0$, for all $y \in K$.

Proof. Following the lines of proof given by Chen [9], one can easily prove. 
Theorem 10. Let $X$ be real reflexive Banach space, and $Y$ be a Banach space. Let $K \subset X$ be a nonempty, bounded, closed, and convex subset of $X$. Let $P: K \rightarrow 2^{Y}$ be such that for each $x \in K, P(x)$ is a proper, closed, convex cone with int $P(x) \neq \varnothing$. Let $f: K \times K \rightarrow Y$ be $P_{-}$-convex and upper semicontinuous in the first and second arguments, respectively, with the condition $f(x, x)=0$ for all $x \in K$. Let conditions (i)-(iii) of Lemma 9 be satisfied and suppose the following conditions hold:

(i) $\eta: K \times K \rightarrow X$ is lower semicontinuous in the second argument;

(ii) $\alpha: X \times X \rightarrow Y$ is weakly lower semicontinuous and $P_{-}$-convex in the first and second arguments, respectively.

Then there exist $x \in K$, such that

$$
\langle T x, \eta(y, x)\rangle+f(y, x) \Varangle_{\text {int } P(x)} 0, \quad \forall y \in K .
$$

Proof. Define two set-valued mappings $F, G: K \rightarrow 2^{K}$ as follows:

$$
\begin{aligned}
F(y)= & \{x \in K:\langle T x, \eta(y, x)\rangle \\
& \left.+f(y, x) \Varangle_{\operatorname{int} P(x)} 0\right\}, \quad \forall y \in K, \\
G(y)= & \{x \in K:\langle T y, \eta(y, x)\rangle \\
& \left.+f(y, x)-\alpha(x, y) \Varangle_{\operatorname{int} P(x)} 0\right\}, \quad \forall y \in K .
\end{aligned}
$$

Then $F(y)$ and $G(y)$ are nonempty since $y \in G(y) \cap F(y)$. We claim that $F$ is a KKM mapping. If this is not true, then there exist a finite set $\left\{y_{1}, \ldots, y_{n}\right\} \subset K$ and $t_{i} \geq 0, i=1, \ldots, n$ with $\sum_{i=1}^{n} t_{i}=1$ such that $y=\sum_{i=1}^{n} t_{i} y_{i} \notin \bigcup_{i=1}^{n} F\left(y_{i}\right)$. Now by the definition of $F$, we have

$$
\left\langle T y, \eta\left(y_{i}, y\right)\right\rangle+f\left(y_{i}, y\right) \leq_{\text {int } P(y)} 0, \quad i=1, \ldots, n .
$$

Now we have

$$
\begin{aligned}
0 & =\langle T y, \eta(y, y)\rangle+f(y, y) \\
& =\left\langle T y, \eta\left(\sum_{i=1}^{n} t_{i} y_{i}, y\right)\right\rangle+f\left(\sum_{i=1}^{n} t_{i} y_{i}, y\right) \\
& =\sum_{i=1}^{n} t_{i}\left[\left\langle T y, \eta\left(y_{i}, y\right)\right\rangle+f\left(y_{i}, y\right)\right] \\
& \leq_{\text {int } P(y)} 0,
\end{aligned}
$$

which leads to a contradiction since $P$ is a proper cone. Thus our claim is verified. So $F$ is a KKM mapping.

Now we prove $F(y) \subset G(y)$ for every $y \in K$. Indeed let $x \in F(y)$. Then, we have

$$
\langle T x, \eta(y, x)\rangle+f(y, x) \Varangle_{\text {int } P(x)} 0 .
$$

Since $T$ is generalized relaxed monotone, we have

$$
\begin{aligned}
& \langle T y, \eta(y, x)\rangle+f(y, x) \\
& \quad \geq_{P_{-}}\langle T x, \eta(y, x)\rangle+f(y, x)+\alpha(x, y) .
\end{aligned}
$$

From Lemma 6, we have

$$
\langle T y, \eta(y, x)\rangle+f(y, x)-\alpha(x, y) \Varangle_{\mathrm{int} P(x)} 0,
$$

that is, $x \in G(y)$, which follows $F(y) \subset G(y)$, for each $y \in K$ and so $G$ is also a KKM mapping. Now we claim that for each $y \in K, G(y) \subset K$ is closed in the weak topology of $X$.

Indeed suppose $\bar{x} \in \overline{G(y)}^{w}$, the weak closure of $G(y)$. Since $X$ is reflexive, there is a sequence $\left\{x_{n}\right\}$ in $G(y)$ such that $\left\{x_{n}\right\}$ converges weakly to $\bar{x} \in K$. Then

$$
\left\langle T y, \eta\left(y, x_{n}\right)\right\rangle+f\left(y, x_{n}\right)-\alpha\left(x_{n}, y\right) \Varangle_{\operatorname{int} P\left(x_{n}\right)} 0,
$$

which implies that

$$
\left\langle T y, \eta\left(y, x_{n}\right)\right\rangle+f\left(y, x_{n}\right)-\alpha\left(x_{n}, y\right) \in Y \backslash\left\{-\operatorname{int} P\left(x_{n}\right)\right\} .
$$

Since $\eta(y, \cdot)$ and $f(y, \cdot)$ are lower and upper semicontinuous and $\alpha(\cdot, y)$ and $Y \backslash\left\{-\operatorname{int} P\left(x_{n}\right)\right\}$ are weakly lower semicontinuous, therefore

$$
\langle T y, \eta(y, \bar{x})\rangle+f(y, \bar{x})-\alpha(\bar{x}, y) \in Y \backslash\{-\operatorname{int} P(\bar{x})\} .
$$

Thus we get

$$
\langle T y, \eta(y, \bar{x})\rangle+f(y, \bar{x})-\alpha(\bar{x}, y) \Varangle_{\text {int } P(\bar{x})} 0
$$

and so $\bar{x} \in G(y)$. This shows that $G(y)$ is weakly closed for each $y \in K$. Our claim is then verified. Since $X$ is reflexive and $K \subset X$ is nonempty, bounded, closed, and convex, $K$ is a weakly compact subset of $X$ and so $G(y)$ is also weakly compact. According to Lemma 7,

$$
\bigcap_{y \in K} G(y) \neq \varnothing \text {. }
$$

This implies that there exists $x \in K$ such that

$$
\langle T y, \eta(y, x)\rangle+f(y, x)-\alpha(x, y) \Varangle_{\text {int } P(x)} 0, \quad \forall y \in K .
$$

Therefore by Minty's type Lemma 9, we conclude that there exist $x \in K$ such that

$$
\langle T x, \eta(y, x)\rangle+f(y, x) \not_{\operatorname{int} P(x)} 0, \quad \forall y \in K .
$$

This completes the proof.

\section{Main Results}

Throughout this section, let $X$ be real reflexive Banach space, $Y$ a Banach space. Let $K \subset X$ be a nonempty, bounded, closed, and convex subset of $X$. Let $P: K \rightarrow 2^{Y}$ be such that for each $x \in K, P(x)$ is a proper, closed, convex cone with int $P(x) \neq \varnothing$ and let $P_{-}=\bigcap_{x \in K} P(x)$.

Some nonlinear mapping consisting with two variables, may be monotone with respect to the first variable and compact with respect to the second one. However, we cannot always expect for them to be monotone or compact with respect to the two variables simultaneously. Keeping this complexity in mind we are interested in the so called semimonotone mapping. We now give the concept of a generalized relaxed semimonotone mapping. 
Definition 11. Let $K \subset X$ be a nonempty, closed, and convex subset of $X$. Let $\eta: K \times K \rightarrow X$ be a mapping and let $\alpha: X \times X \rightarrow Y$ be a bifunction such that $\lim _{t \rightarrow 0^{+}}(\alpha(x, t y+$ $(1-t) x) / t)=0$ and $f: K \times K \rightarrow Y$. A mapping $A: K \times K \rightarrow L(X, Y)$ is said to be generalized relaxed semimonotone mapping, if the following conditions hold:

(i) for every $u \in K, A(u, \cdot)$ is a generalized relaxed monotone mapping, that is,

$$
\langle A(u, y)-A(u, x), \eta(y, x)\rangle-\alpha(x, y) \geq_{P_{-}} 0, \quad \forall x, y \in K
$$

(ii) for every $y \in K, A(\cdot, y)$ is completely continuous, that is, when $u_{n} \rightarrow{ }^{w} u, A\left(u_{n}, y\right) \rightarrow A(u, y)$ (by the norm of operators), where $\rightarrow$ denotes the weak convergence.

Remark 12. When $Y=\mathbb{R}, P(x)=\mathbb{R}^{+}$, and $\eta(y, x)=y-$ $x$, for all $x, y \in K$ and $\alpha \equiv 0$, then this is exactly the concept which was introduced by Chen [9].

Example 13. Let $X=Y=\mathbb{R}^{2}, K=[0,1] \times[0,1]$. Let $P$ : $K \rightarrow 2^{Y}$ be defined by

$$
P(x)=\left\{\left(y_{1}, y_{2}\right) \in Y \mid y_{1} \geq 0, y_{2} \geq 0\right\}, \quad x \in K .
$$

So

$$
P_{-}=\bigcap_{x \in K} P(x)=\left\{\left(y_{1}, y_{2}\right) \in Y \mid y_{1} \geq 0, y_{2} \geq 0\right\}
$$

Let $T: K \rightarrow L(X, Y)$ be defined by

$$
T(x)=\left(\begin{array}{cc}
x_{1} & x_{2} \\
x_{1}+1 & x_{2}+1
\end{array}\right), \quad \forall x=\left(x_{1}, x_{2}\right) \in K .
$$

Also $\eta: K \times K \rightarrow X, \alpha: X \times X \rightarrow Y$ are define by $\eta(y, x)=$ $y-x$, for all $x, y \in K$ and $\alpha(x, y)=(x-y)^{2}$, for all $x, y \in X$, respectively.

Let us suppose $A: K \times K \rightarrow L(X, Y)$ is defined by

$$
\begin{array}{r}
A(x, y)=\left(\begin{array}{cc}
x_{1}+y_{1} & x_{2}+y_{2} \\
y_{1} & y_{2}
\end{array}\right), \quad \forall x=\left(x_{1}, x_{2}\right), \\
y=\left(y_{1}, y_{2}\right) \in K .
\end{array}
$$

First, we show that $A$ is a generalized relaxed monotone mapping. Indeed, for each $u=\left(u_{1}, u_{2}\right), v=\left(v_{1}, v_{2}\right) \in K$, it follows that

$$
\begin{aligned}
& \langle A(u, y)-A(v, y), \eta(u-v)\rangle-\alpha(v, u) \\
& =\left(\begin{array}{ll}
u_{1}-v_{1} & u_{2}-v_{2} \\
u_{1}-v_{1} & u_{2}-v_{2}
\end{array}\right)\left(\begin{array}{l}
u_{1}-v_{1} \\
u_{2}-v_{2}
\end{array}\right)-\left(\begin{array}{l}
\left(v_{1}-u_{1}\right)^{2} \\
\left(v_{2}-u_{2}\right)^{2}
\end{array}\right) \\
& =\left(\begin{array}{c}
\left(u_{2}-v_{2}\right)^{2} \\
\left(u_{1}-v_{1}\right)^{2}
\end{array}\right) \geq_{P_{-}} 0 .
\end{aligned}
$$

The norm of $A$ is defined as $\|A\|=|x+y|+|y|$, for all $x=$ $\left(x_{1}, x_{2}\right)$, and $y=\left(y_{1}, y_{2}\right) \in K$.
Now, for fixed $v \in K$, if $u_{n} \in K, u \in K$, and $u_{n} \rightarrow{ }^{w} u$, it is easy to prove that

$$
\left\|A\left(u_{n}, v\right)-A(u, v)\right\| \longrightarrow 0
$$

Hence, for every $v \in K, A(\cdot, v)$ is completely continuous. Therefore, the mapping defined as above is a generalized relaxed semimonotone mapping.

Now we will pose the main problem of our study. In this paper, we investigate the following generalized vector variational-like inequality problem (for short, GVVLIP) is to find a vector $u \in K$ satisfying

$$
\langle A(u, u), \eta(v, u)\rangle+f(v, u) \$_{\operatorname{int} P(u)} 0, \quad \forall v \in K,
$$

where $A: K \times K \rightarrow L(X, Y)$ is a nonlinear mapping and $\eta: K \times K \rightarrow X, f: K \times K \rightarrow Y$ are the two vector-valued bimappings.

The GVVLIP (31) includes many variational inequality problems as special cases.

Some special cases of GVVLIP (31) are as follows.

(I) If $\eta(v, u)=v-u$ and $f \equiv 0$, then the GVVLIP (31) reduces to the following variational inequality problem of finding $u \in K$ such that

$$
\langle A(u, u), v-u\rangle \Varangle_{\text {int } P(u)} 0, \quad \forall v \in K,
$$

which was introduced and studied by Zheng [7]

(II) If $Y=\mathbb{R}, P(u)=[0,+\infty)$, for all $u \in K$. Also if $\eta(v, u)=v-u, f \equiv 0$ and $L(X, Y)$ is the dual space $X^{*}$ of $X$, then the GVVLIP (31) reduces to the following variational inequality problem of finding $u \in K$ such that

$$
\langle A(u, u), v-u\rangle \geq 0, \quad \forall v \in K
$$

which was introduced and investigated by Chen [9]. He obtained some existence results and discussed their applications in partial differential equations of divergence form.

We recall the following fixed-point theorem, by Zeidle [16], which will play an important role in establishing our existing results for GVVLIP (31).

Theorem 14 (see [16]). The set-valued mapping $F: M \rightarrow$ $2^{M}$ has a fixed point, if the following conditions are satisfied:

(1) $M$ is compact, convex, and nonempty set in locally convex space;

(2) $F(x)$ is convex, closed, and nonempty for every $x \in M$;

(3) $F$ is upper semicontinuous on $M$.

Now, we have the following existence results for GVVLIP (31) involving a generalized relaxed semimonotone mapping in reflexive Banach spaces.

Theorem 15. Let $X$ be real reflexive Banach space, and let $Y$ be a Banach space. Let $K \subset X$ be a nonempty, bounded, 
closed, and convex subset of $X$. Let $P: K \rightarrow 2^{Y}$ be such that for each $x \in K, P(x)$ is a proper, closed, convex cone with int $P(x) \neq \varnothing$. Let $A: K \times K \rightarrow L(X, Y)$ be a generalized relaxed semimonotone mapping. Suppose the following conditions hold:

(i) $f: K \times K \rightarrow Y$ is $P_{-}$-convex with $f(x, x)=$ 0 , for all $x \in K$ and upper semicontinuous in the second argument;

(ii) $\eta: K \times K \rightarrow X$ is an affine mapping with $\eta(x, y)+$ $\eta(y, x)=0$, for all $x, y \in K$ and lower semicontinuous in the second argument;

(iii) The set-valued mapping $W: K \rightarrow 2^{Y}$ defined as $W(x)=Y \backslash\{-\operatorname{int} P(x)\}$, for all $x \in K$, is weakly upper semicontinuous and concave;

(iv) $\alpha: X \times X \rightarrow Y$ is $P_{-}$-concave and weakly lower semicontinuous in the second and first argument, respectively;

(v) For each fixed $v \in K, A(v, \cdot): K \times K \rightarrow L(X, Y)$ is continuous on each finite dimensional subspace of $X$.

Then there exists a $u_{0} \in K$ such that

$$
\left\langle A\left(u_{0}, u_{0}\right), \eta\left(v, u_{0}\right)\right\rangle+f\left(v, u_{0}\right) \not_{\text {int } P\left(u_{0}\right)} 0, \quad \forall v \in K .
$$

Proof. Let $F$ be a finite dimensional subspace of $X$ and $K_{F}=$ $K \bigcap F \neq \varnothing$. For each $v \in K_{F}$, we consider the following generalized vector variational-like inequality problem. Find $u_{0} \in K_{F}$ such that

$$
\left\langle A\left(v, u_{0}\right), \eta\left(u, u_{0}\right)\right\rangle+f\left(u, u_{0}\right) \Varangle_{\text {int } P\left(u_{0}\right)} 0, \quad \forall u \in K_{F} .
$$

Since $K_{F} \subset F$ is bounded, closed, and convex, $A(v, \cdot)$ is continuous on $K_{F}$ and generalized relaxed monotone for each fixed $v \in K$. From Theorem 10, we know our problem has solution $u_{0} \in K_{F}$.

Define a set-valued mapping $T: K_{F} \rightarrow 2^{K_{F}}$ as follows:

$$
\begin{aligned}
F(v)= & \left\{w \in K_{F}:\langle A(v, w), \eta(u, w)\rangle\right. \\
& \left.+f(u, w) \nless_{\operatorname{int} P(w)} 0, \forall u \in K_{F}\right\} .
\end{aligned}
$$

It follows from Minty's type Lemma 9, that for each fixed $v \in$ $K_{F}$

$$
\begin{aligned}
\{w \in & K_{F}:\langle A(v, w), \eta(u, w)\rangle \\
+ & \left.f(u, w) \Varangle_{\operatorname{int} P(w)} 0, \forall u \in K_{F}\right\} \\
= & \left\{w \in K_{F}:\langle A(v, u), \eta(u, w)\rangle\right. \\
& \left.\quad+f(u, w)-\alpha(w, u) \Varangle_{\operatorname{int} P(w)} 0, \forall u \in K_{F}\right\} .
\end{aligned}
$$

Now we will use the fixed-point theorem to verify the existence of the solution of the problem in a finite dimensional.
Since $F$ is of finite dimensional, hence $K_{F}$ is compact. First, we claim that

$$
\begin{array}{r}
F(v)=\left\{w \in K_{F}:\langle A(v, u), \eta(u, w)\rangle+f(u, w)\right. \\
-\alpha(w, u) \in Y \backslash\{-\operatorname{int} P(w)\}=W(w)\}
\end{array}
$$

is convex. Indeed, let $w_{1}, w_{2} \in F(v)$ and $m, n \geq 0$, such that $m+n=1$

$$
\begin{aligned}
& m\left[\left\langle A(v, u), \eta\left(u, w_{1}\right)\right\rangle+f\left(u, w_{1}\right)-\alpha\left(w_{1}, u\right)\right]=m W\left(w_{1}\right), \\
& n\left[\left\langle A(v, u), \eta\left(u, w_{2}\right)\right\rangle+f\left(u, w_{2}\right)-\alpha\left(w_{2}, u\right)\right]=m W\left(w_{2}\right) .
\end{aligned}
$$

Since $\eta(u, \cdot)$ is affine and $f(u, \cdot), \alpha(\cdot, u)$ are $P_{-}$-convex, then from preceding two inclusions we have

$$
\begin{aligned}
& \left\langle A(v, u), \eta\left(u, m w_{1}+n w_{2}\right)\right\rangle+f\left(u, m w_{1}+n w_{2}\right) \\
& \quad-\alpha\left(\left(m w_{1}+n w_{2}\right), u\right) \in m W\left(w_{1}\right)+n W\left(w_{2}\right) .
\end{aligned}
$$

Since $W$ is concave, we have $m w_{1}+n w_{2} \in F(v)$, that is, $F(v)$ is convex and our claim is then verified. Now, we claim that $F(v)$ is closed. Let $w_{j} \in F(v)$ such that $w_{j} \rightarrow w$, then

$$
\begin{gathered}
\left\langle A(v, u), \eta\left(u, w_{j}\right)\right\rangle+f\left(u, w_{j}\right)-\alpha\left(w_{j}, u\right) \\
\in Y \backslash\left\{-\operatorname{int} P\left(w_{j}\right)\right\}, \\
\left\langle A(v, u), \eta\left(u, w_{j}\right)\right\rangle+f\left(u, w_{j}\right)-\alpha\left(w_{j}, u\right) \in W\left(w_{j}\right) .
\end{gathered}
$$

Since $f(y, \cdot)+\alpha(\cdot, y)$ is upper semicontinuous, also $A(v, u) \in$ $L(X, Y)$ and $W$ is upper semicontinuous; therefore

$$
\begin{aligned}
& \left\langle A(v, u), \eta\left(u, w_{j}\right)\right\rangle+f\left(u, w_{j}\right)-\alpha\left(w_{j}, u\right) \\
& \quad \longrightarrow\langle A(v, u), \eta(u, w)\rangle+f(u, w)-\alpha(w, u) \in W(w) .
\end{aligned}
$$

This implies $w \in F(v)$; hence $F(v)$ is closed. Next, we claim that $F$ is upper semicontinuous. Let $v_{j} \rightarrow v, w_{j} \rightarrow F\left(v_{j}\right)$, and $w_{j} \rightarrow w$, then we have

$$
\left\langle A\left(v_{j}, u\right), \eta\left(u, w_{j}\right)\right\rangle+f\left(u, w_{j}\right)-\alpha\left(w_{j}, u\right) \in W\left(w_{j}\right) .
$$

From the complete continuity of $A(\cdot, u)$ and the lower semicontinuity of $\eta(u, \cdot)$, we have

$$
\left\langle A\left(v_{j}, u\right), \eta\left(u, w_{j}\right)\right\rangle \longrightarrow\langle A(v, u), \eta(u, w)\rangle
$$

which implies that

$$
\langle A(v, u), \eta(u, w)\rangle+f(u, w)-\alpha(w, u) \in W(w),
$$

that is, $w \in F(v)$; thus our claim is then verified. Hence $F$ is upper semicontinuous. By Fan-Glicksberg fixed-point 
theorem, there exists a $v_{0} \in F\left(v_{0}\right)$ that is, there exists a $v_{0} \in K_{F}$ such that

$$
\left\langle A\left(v_{0}, v_{0}\right), \eta\left(u, v_{0}\right)\right\rangle+f\left(u, v_{0}\right) \Varangle_{\text {int } P\left(v_{0}\right)} 0, \quad \forall u \in K_{F} .
$$

Now we generalize this result to whole space.

Let $\Omega \equiv\{F \subset X: F$ be finite dimensional, $F \bigcap K \neq \varnothing\}$ and let $\Gamma_{F} \equiv\{w \in K:\langle A(w, u), \eta(u, w)\rangle+f(u, w)-$ $\alpha(w, u) \Varangle_{\text {int } P(w)} 0$, for all $\left.u \in K_{F}\right\}, F \in \Omega$.

From the above we know that for all $F \in \Omega, \Gamma_{F} \neq \varnothing$. Let $\overline{\Gamma_{F}{ }^{w}}$ denote the weak closure of $\Gamma_{F}$. For any $F_{i} \in \Omega, i=$ $1, \ldots, n$, we know that $\Gamma_{\bigcup_{i=1}^{n} F_{i}} \subseteq \bigcap_{i=1}^{n} \Gamma_{F_{i}} \subseteq \bigcap_{i=1}^{n}{\overline{\Gamma_{F}}}^{w}$. Therefore, $\bigcap_{i=1}^{n}{\overline{\Gamma_{F_{i}}}}^{w} \neq \varnothing$. Since $K$ is weakly compact, from the finite intersection property, we have $\bigcap_{x \in \Omega}{\overline{\Gamma_{F}}}^{w} \neq \varnothing$. Let $w_{0} \in \bigcap_{x \in \Omega}{\overline{\Gamma_{F}}}^{w}$, we see that

$$
\left\langle A\left(w_{0}, w_{0}\right), \eta\left(u, w_{0}\right)\right\rangle+f\left(u, w_{0}\right) \Varangle_{\text {int } P\left(w_{0}\right)} 0 .
$$

Indeed, for each $u \in K$, let $F \in \Omega$, such that $u \in K_{F}, w_{0} \in K_{F}$. From $w_{0} \in{\overline{\Gamma_{F}}}^{w}$, there exists $w_{j} \in \Gamma_{F}$, that is,

$$
\begin{gathered}
\left\langle A\left(w_{j}, u\right), \eta\left(u, w_{j}\right)\right\rangle+f\left(u, w_{j}\right)-\alpha\left(w_{j}, u\right) \\
\in Y \backslash\left\{-\operatorname{int} P\left(w_{j}\right)\right\} \in W\left(w_{j}\right),
\end{gathered}
$$

such that $w_{j} \rightarrow{ }^{w} w_{0}$, from the complete continuity of $A(\cdot, v)$, upper semicontinuity of $f(u, \cdot)$, and lower semicontinuity of $\alpha(\cdot, u)$, we have

$$
\left\langle A\left(w_{0}, u\right), \eta\left(u, w_{0}\right)\right\rangle+f\left(u, w_{0}\right)-\alpha\left(w_{0}, u\right) \in W\left(w_{0}\right) .
$$

From Lemma 9, we have

$$
\left\langle A\left(w_{0}, w_{0}\right), \eta\left(u, w_{0}\right)\right\rangle+f\left(u, w_{0}\right) \Varangle_{\text {int } P\left(w_{0}\right)} 0, \quad \forall u \in K .
$$

This completes the proof.

If the boundedness of $K$ is dropped off, then we have the following theorem under certain coercivity condition.

Theorem 16. Let $X$ be real reflexive Banach space and $Y$ be a Banach space. Let $0 \in K \subset X$ be a nonempty, closed, and convex subset of $X$. Let $P: K \rightarrow 2^{Y}$ be such that for each $x \in$ $K, P(x)$ is a proper, closed, convex cone with int $P(x) \neq \varnothing$. Let $A: K \times K \rightarrow L(X, Y)$ be a generalized relaxed semimonotone mapping. Let conditions ( $(i)-(v)$ of Theorem 15 be satisfied and

$$
\lim _{\|u\| \rightarrow \infty}\langle A(u, u), \eta(u, 0)\rangle+f(0, u) \geq_{\operatorname{int} P(u)} 0
$$

then there exist $u \in K$ such that

$$
\langle A(u, u), \eta(v, u)\rangle+f(v, u) \nless_{\operatorname{int} P(u)} 0, \quad \forall v \in K .
$$

Proof. For each $r>0$, let $B[0, r]$ denote the closed ball in the Banach space $X$ with center 0 and radius $r$. By Theorem 15, for each $r \in \mathbb{N}$, there exists $u_{r} \in B[0, r] \cap K$ such that

$$
\begin{array}{r}
\left\langle A\left(u_{r}, u_{r}\right), \eta\left(v, u_{r}\right)\right\rangle+f\left(v, u_{r}\right) \not_{\operatorname{int} P\left(u_{r}\right)} 0, \\
\forall v \in B[0, r] \cap K .
\end{array}
$$

Since $0 \in K$, we have

$$
\left\langle A\left(u_{r}, u_{r}\right), \eta\left(0, u_{r}\right)\right\rangle+f\left(0, u_{r}\right) \Varangle_{\text {int } P\left(u_{r}\right)} 0 .
$$

From condition (vi), we know that $\left\{u_{r}\right\}$ is bounded. So, we may suppose that $u_{r}$ converges to $u$ as $r \rightarrow \infty$. It follows from Lemma 9, that

$$
\begin{array}{r}
\left\langle A\left(u_{r}, v\right), \eta\left(v, u_{r}\right)\right\rangle+f\left(v, u_{r}\right)-\alpha\left(u_{r}, v\right) \Varangle_{\text {int } P\left(u_{r}\right)} 0, \\
\forall v \in K \cap B_{r} .
\end{array}
$$

Since $A$ is completely continuous, $\eta, \alpha$ are lower semicontinuous, and $f, W$ are upper semicontinuous. Therefore, it follows that

$$
\begin{array}{r}
\langle A(u, v), \eta(v, u)\rangle+f(v, u)-\alpha(u, v) \not_{\text {int } P(u)} 0, \\
\forall v \in K \cap B_{r} .
\end{array}
$$

Again from Lemma 9, we get

$$
\langle A(u, u), \eta(v, u)\rangle+f(v, u) \Varangle_{\text {int } P(u)} 0, \quad \forall v \in K .
$$

This completes the proof.

Remark 17. Theorems 15 and 16 improve and generalize Theorems 3.1 and 3.2 of Fang and Huang [10] and Theorem 2.1 to Theorem 2.6 of Chen [9].

\section{References}

[1] F. Giannessi, "Theorems of alternative, quadratic programs and complementarity problems," in Variational Inequalities and Complementarity Problems, R. W. Cottle, F. Giannessi, and J. L. Lions, Eds., pp. 151-186, John Wiley \& Sons, New York, NY, USA, 1980

[2] G. Y. Chen, "Existence of solutions for a vector variational inequality: an extension of the Hartmann-Stampacchia theorem," Journal of Optimization Theory and Applications, vol. 74, no. 3, pp. 445-456, 1992.

[3] G. Y. Chen and X. Q. Yang, “The vector complementary problem and its equivalences with the weak minimal element in ordered spaces," Journal of Mathematical Analysis and Applications, vol. 153, no. 1, pp. 136-158, 1990.

[4] F. Giannessi, Vector Variational Inequalities and Vector Equilibrium, Kluwer Academic Press, 1999.

[5] K. L. Lin, D. P. Yang, and J. C. Yao, "Generalized vector variational inequalities," Journal of Optimization Theory and Applications, vol. 92, no. 1, pp. 117-126, 1997.

[6] X. Q. Yang, "Vector variational inequality and its duality," Nonlinear Analysis, vol. 21, no. 11, pp. 869-877, 1993.

[7] F. Zheng, "Vector variational inequalities with semi-monotone operators," Journal of Global Optimization, vol. 32, no. 4, pp. 633-642, 2005.

[8] F. E. Browder, "Semi-contract and semi-accretive nonlinear mappings in Banach space," Bulletin of the American Mathematical Society, vol. 74, pp. 660-665, 1968.

[9] Y. Q. Chen, "On the semi-monotone operator theory and applications," Journal of Mathematical Analysis and Applications, vol. 231, no. 1, pp. 177-192, 1999. 
[10] Y. P. Fang and N. J. Huang, "Variational-like inequalities with generalized monotone mappings in Banach spaces," Journal of Optimization Theory and Applications, vol. 118, no. 2, pp. 327-338, 2003.

[11] F. Usman and S. A. Khan, "A generalized mixed vector variational-like inequality problem," Nonlinear Analysis, vol. 71, no. 11, pp. 5354-5362, 2009.

[12] D. Goeleven and D. Motreanu, "Eigenvalue and dynamic problems for variational and hemivariational inequalities," Communications on Applied Nonlinear Analysis, vol. 3, no. 4, pp. 1-21, 1996.

[13] R. U. Verma, "On monotone nonlinear variational inequality problems," Commentationes Mathematicae Universitatis Carolinae, vol. 39, no. 1, pp. 91-98, 1998.

[14] R. U. Verma, "Nonlinear variational inequalities on convex subsets of banach spaces," Applied Mathematics Letters, vol. 10, no. 4, pp. 25-27, 1997.

[15] K. Fan, "A generalization of Tychonoff's fixed point theorem," Mathematische Annalen, vol. 142, no. 3, pp. 305-310, 1961.

[16] E. Zeidle, Nonlinear Functional Analysis and Its Applications, vol. 4, Springer, Berlin, Germany, 1993. 


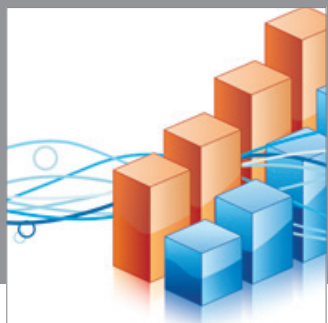

Advances in

Operations Research

mansans

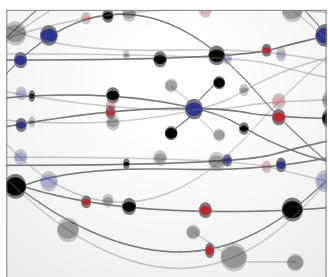

The Scientific World Journal
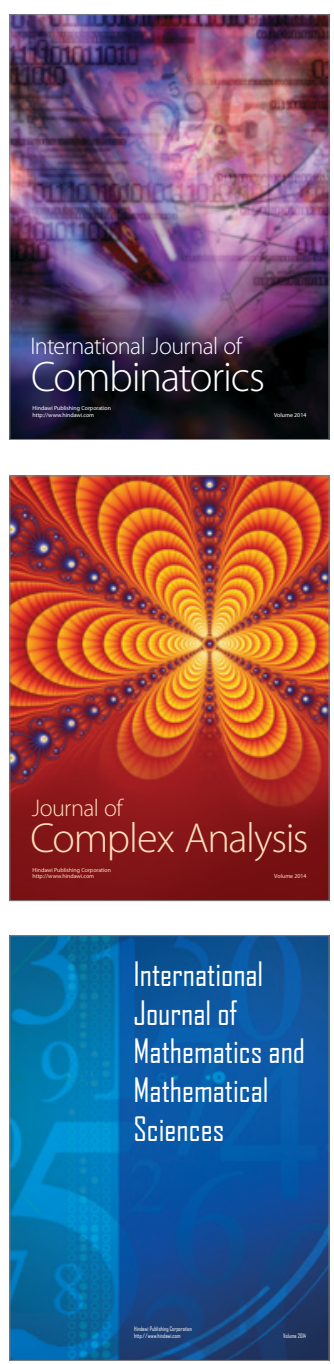
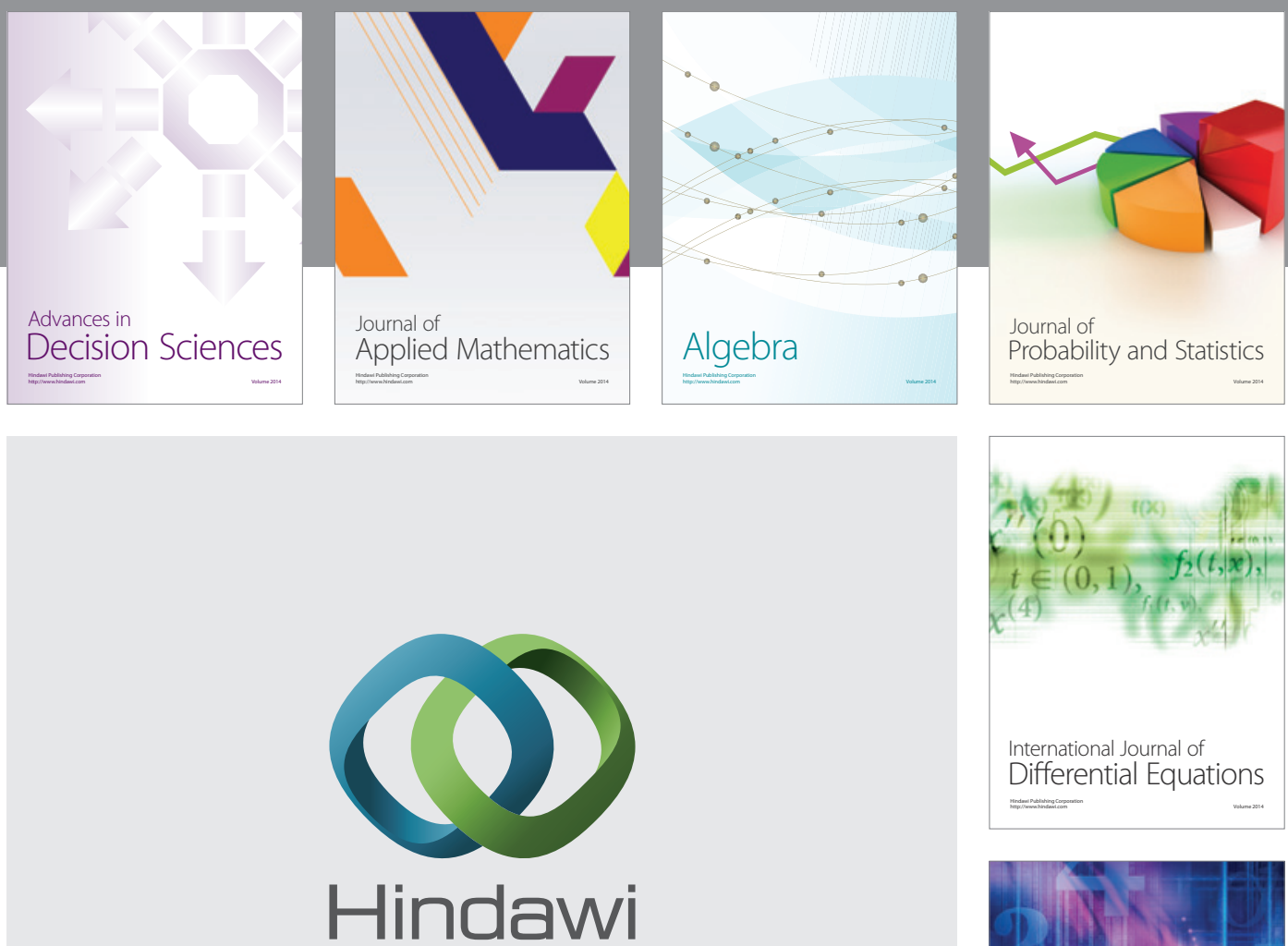

Submit your manuscripts at http://www.hindawi.com
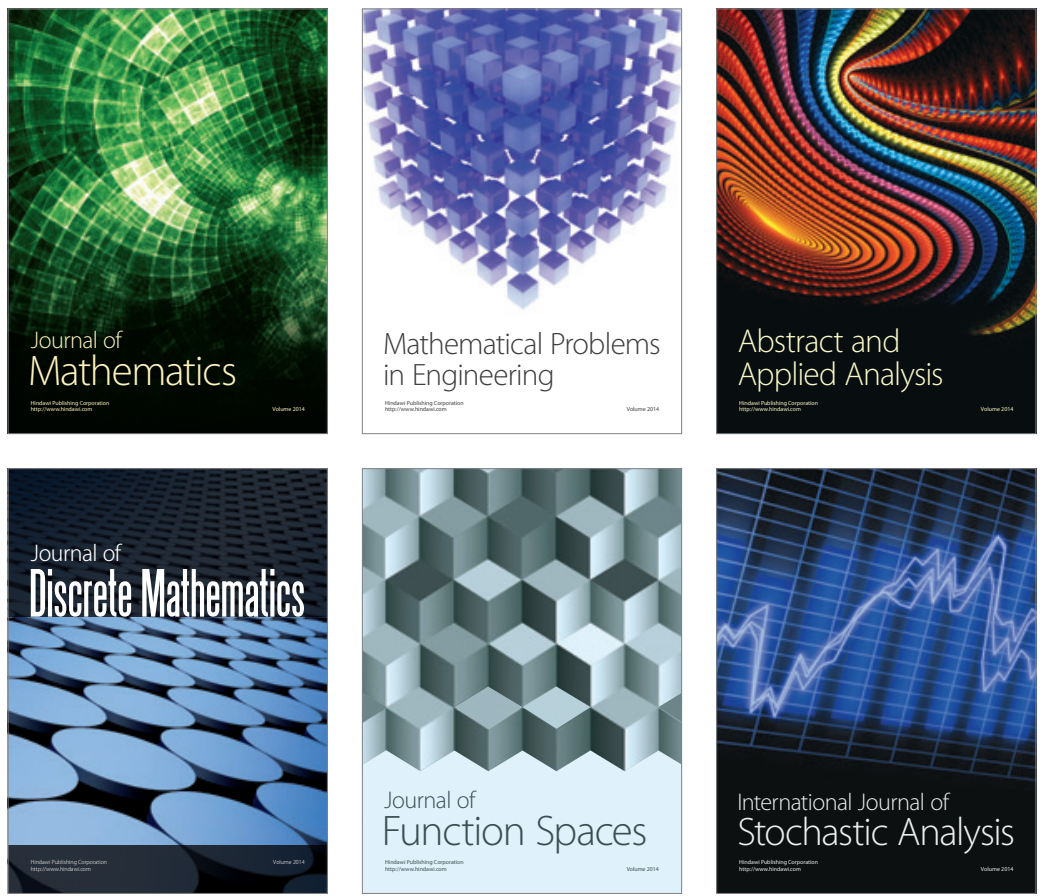

Journal of

Function Spaces

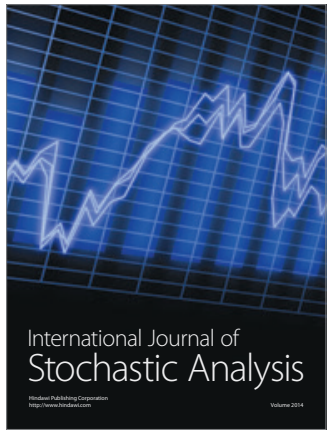

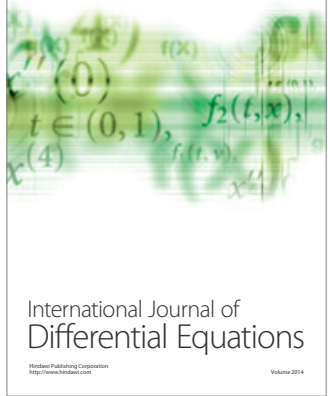
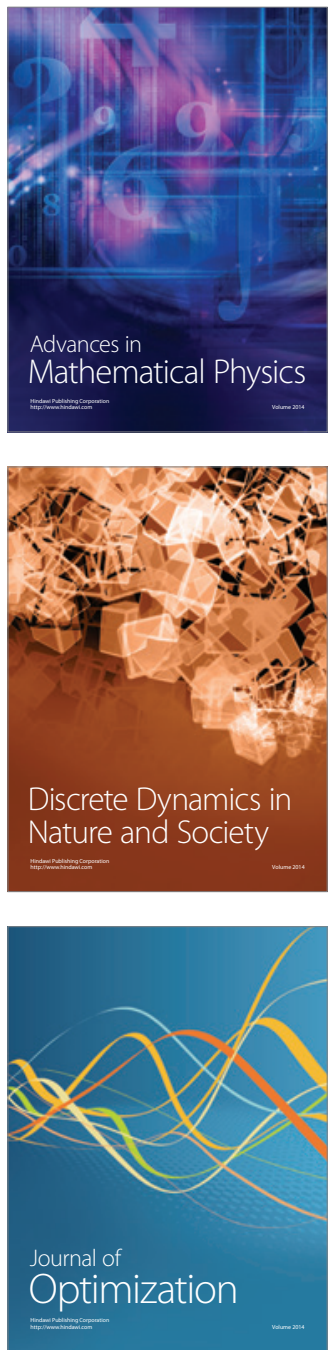